\title{
Stress doses of hydrocortisone reduce chronic stress symptoms and improve health-related quality of life in high-risk patients after cardiac surgery: A randomized study
}

Florian Weis, MD, ${ }^{a}$ Erich Kilger, MD, PhD, ${ }^{a}$ Benno Roozendaal, $\mathrm{PhD},{ }^{\mathrm{b}}$ Dominique J.-F. de Quervain, MD, PhD, Peter Lamm, MD, PhD, ${ }^{d}$ Michael Schmidt, MD, ${ }^{e}$ Martin Schmölz, MD, ${ }^{a}$ Josef Briegel, MD, PhD, ${ }^{a}$ and Gustav Schelling, MD, PhD

丹 Supplemental material is available online.
From the Departments of Anesthesiology ${ }^{\mathrm{a}}$ and Cardiac Surgery ${ }^{\mathrm{d}}$ and the Institute for Medical Informatics, Biometry and Epidemiology, ${ }^{\mathrm{e}}$ Ludwig-Maximilians-University, Munich, Germany; the Center for the Neurobiology of Learning and Memory, ${ }^{\text {b }}$ Department of Neurobiology and Behavior, University of California, Irvine, Calif; and the Division of Psychiatry Research, ${ }^{\mathrm{c}}$ University of Zurich, Zurich, Switzerland.

Received for publication April 7, 2005; revisions received June 28, 2005; accepted for publication July 12, 2005.

Address for reprints: Gustav Schelling, $\mathrm{MD}, \mathrm{PhD}$, Ludwig-Maximilians University, Klinikum Grosshadern, Department of Anaesthesiology, 81377 Muenchen, Germany (E-mail: gustav.schelling@med. uni-muenchen.de).

J Thorac Cardiovasc Surg 2006;131:277-82 $0022-5223 / \$ 32.00$

Copyright () 2006 by The American Association for Thoracic Surgery

doi:10.1016/j.jtcvs.2005.07.063
Objectives: Improvement in health-related quality of life is a major object of cardiac surgery. However, high stress exposure during the perioperative period of cardiac surgery can result in the formation of traumatic memories and symptoms of chronic stress or even posttraumatic stress disorder, which can have negative effects on health-related quality-of-life outcome. In this controlled study we examined whether exogenously administered stress doses of hydrocortisone during cardiac surgery reduce perioperative stress exposure and the long-term incidence of chronic stress symptoms and improve health-related quality of life after cardiac surgery.

Methods: Thirty-six high-risk patients undergoing cardiac surgery were prospectively randomized to receive either stress doses of hydrocortisone or placebo. Of 28 available patients at 6 months after cardiac surgery, 14 had received hydrocortisone, and 14 had received placebo. Traumatic memories, chronic stress symptoms (posttraumatic stress disorder scores), and health-related quality of life were measured by using validated questionnaires.

Results: Compared with patients from the placebo group, patients from the hydrocortisone group had a significantly shorter duration of intensive care unit treatment, required lower doses of the stress hormone norepinephrine during cardiac surgery, and had significantly fewer stress symptoms and a better health-related quality of life regarding physical function, chronic pain, general health, vitality, and mental health during follow-up. The groups did not differ with regard to the number or type of intensive care unit-related traumatic memories.

Conclusions: The use of stress doses of hydrocortisone in high-risk cardiac surgical patients reduces perioperative stress exposure, decreases chronic stress symptoms, and improves health-related quality of life at 6 months after cardiac surgery.

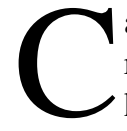
ardiac surgery (CS) with cardiopulmonary bypass (CPB) improves healthrelated quality of life (HRQL) in the majority of patients. ${ }^{1}$ On the other hand, consequences of CS also include a considerable risk for the development of postoperative organ dysfunction, prolonged treatment in an intensive care unit (ICU), and, in due course, a considerable risk of cognitive decline, which includes deficits in verbal and visual memory, attention, and concentration. ${ }^{2}$ In addition, CS represents a high stress exposure that is the combined result of critical illness, CPB, and major operations; exogenously administered adrenergic stress hormones; and traumatic experiences, such as pain, anxiety, or respiratory distress (particularly during ICU treatment). ${ }^{3}$ There is increasing evidence that high perioperative stress exposure in patients undergoing CS might result in chronic stress-related illness and even 


\author{
Abbreviations and Acronyms \\ $\mathrm{CPB}=$ cardiopulmonary bypass \\ $\mathrm{CS}=$ cardiac surgery \\ $\mathrm{HRQL}=$ health-related quality of life \\ ICU = intensive care unit \\ IL-6 = interleukin 6 \\ POD = postoperative day \\ PTSD $=$ posttraumatic stress disorder \\ SF-36 $=$ Short Form-36 \\ TISS $=$ Therapeutic Intervention Scoring System
}

posttraumatic stress disorder (PTSD). ${ }^{3}$ Importantly, the development of chronic stress symptoms or PTSD in patients after CS is associated with a significant decrease in HRQL outcomes, including impairments in physical function and mental health, ${ }^{4}$ which might greatly reduce or even abolish the benefits of $\mathrm{CS}^{3,5}$

In addition to a characteristic pattern of emotional symptoms ${ }^{6}$ and the presence of traumatic memories from highly aversive experiences, ${ }^{3,7}$ many patients with chronic stressrelated illness show distinct neuroendocrine abnormalities, which include increased catecholaminergic activity and, remarkably, a decreased activity of the hypothalamicpituitary-adrenocortical axis, ${ }^{6}$ which is reflected by a reduction in urinary cortisol excretion ${ }^{8}$ or systemic cortisol levels. ${ }^{9}$ Interestingly, there is evidence from 2 recent studies in patients with septic shock ${ }^{10}$ and after $\mathrm{CS}^{11}$ that prolonged glucocorticoid administration (stress doses of hydrocortisone $^{12}$ ) during the actual stress exposure of ICU treatment results in a decreased incidence and intensity of chronic stress symptoms ${ }^{11}$ or PTSD ${ }^{10}$ during follow-up. The abovementioned 2 studies from our group did not investigate other possible long-term consequences of stress doses of hydrocortisone, however, namely a likely improvement in HRQL outcomes that might include both physical and mental aspects. We therefore conducted an additional study in selected patients at high risk for perioperative complications to address this important issue.

\section{Patients and Methods}

Between September 2002 and September 2003, we enrolled 36 high-risk patients in a prospective, randomized, double-blind trial. All patients received a detailed explanation of the purpose of the study and provided written informed consent. The study was approved by the Institutional Review Board of the LudwigMaximilians University of Munich, and data protection met the standard set by German law.

\section{Inclusion and Exclusion Criteria}

The study was performed in high-risk patients undergoing CS with $\mathrm{CPB}$. High risk was defined as a preoperative left ventricular ejection fraction of less than $35 \%$ or an expected duration of $\mathrm{CPB}$ of greater than 97 minutes. ${ }^{13}$ These criteria have been validated in 2 controlled studies by our group ${ }^{11,13}$ and showed that patients who fulfilled these criteria required prolonged ICU treatment and had an increased risk for a severe inflammatory reaction after CPB and an increased incidence of chronic stress symptoms, which were both responsive to stress doses of hydrocortisone. Patients were excluded from the study if they met the following criteria before surgical intervention: pregnancy, emergency operation, hepatic dysfunction (bilirubin $>3 \mathrm{mg} / \mathrm{dL}$ ), renal dysfunction (plasma creatinine $>2 \mathrm{mg} / \mathrm{dL}$ ), a positive serologic test result for HIV, manifest insulin-dependent diabetes mellitus, an extracardial septic focus, chronic or acute inflammatory disease, and inability to provide informed consent. In addition, patients who required glucocorticoids other than hydrocortisone were excluded.

\section{Administration of Study Drugs}

The patients were randomly assigned to one of 2 treatment groups with the use of a computer-generated randomization list. One group of patients received stress doses of hydrocortisone (Pharmacia \& Upjohn, Erlangen, Germany; the hydrocortisone group), ${ }^{12}$ and patients from the other group (the placebo group) received normal saline in identical vials in a double-blind fashion. The vials were prepared by a study nurse who was not involved in the care of patients participating in the trial.

Hydrocortisone administration started with a loading dose (100 mg over 10 minutes administered intravenously) before induction of anesthesia, followed by a continuous infusion of 10 $\mathrm{mg} / \mathrm{h}$ for 24 hours (postoperative day [POD] 1), which was reduced to $5 \mathrm{mg} / \mathrm{h}$ on POD 2 and then tapered to $3 \times 20 \mathrm{mg}$ administered intravenously on POD 3 and $3 \times 10 \mathrm{mg}$ administered intravenously on POD $4 .{ }^{13}$

\section{Measurements and Data Collection}

Because of the established effects of catecholamines on stress responses and stress-influenced memory formation, ${ }^{14}$ the duration of administration and the maximal doses of the stress hormones epinephrine (as an inotropic agent) and norepinephrine (to counteract vasodilatatory hypotension) were registered. In addition, perioperative data collection included the length of mechanical ventilation, peak plasma concentration of lactic acid measured during ICU therapy, serum levels of the proinflammatory cytokine interleukin 6 (IL-6), the Simplified Acute Physiology Score during the first 24 hours in the ICU, the Therapeutic Intervention Scoring System (TISS) score daily during the stay in the ICU (Simplified Acute Physiology Score and TISS scores were used as disease severity indicators; for definition, see Table E1), and length of stay in the ICU.

\section{Evaluation of Study End Points}

At 6 months after CS, the patients were contacted by telephone and received a detailed re-explanation of the purpose of the study. In particular, they were informed that we were interested in their current quality of life, as well as their present state of physical and mental health, without any direct referral to chronic stress symptoms or traumatic memories. After repeated oral consent, the patients received questionnaires for evaluation of HRQL, trau- 
TABLE 1. Comparison of patient characteristics and treatment data between patients from the hydrocortisone and placebo groups

\begin{tabular}{|c|c|c|c|}
\hline Patient and treatment data & Hydrocortisone group $(n=14)$ & Placebo group $(n=14)$ & $P$ value \\
\hline $\operatorname{Sex}(F / M)$ & $4 / 14$ & $5 / 14$ & 1.0 \\
\hline Age $(y)^{*}$ & $68(63-72)$ & $69(63-73)$ & .68 \\
\hline SAPS II*† & $34(30-38)$ & $36(31-46)$ & .51 \\
\hline TISS*† & $99(78-129)$ & $234(149-356)$ & $<.01$ \\
\hline Preoperative left ventricular ejection fraction (\%) & $30(27-40)$ & $30(21-48)$ & .98 \\
\hline Duration of CPB $(\min )^{*}$ & $98(91-154)$ & $112(65-139)$ & .64 \\
\hline Duration of aortic crossclamp $(\mathrm{min})^{*}$ & $75(62-86)$ & $85(62-86)$ & .85 \\
\hline Duration of mechanical ventilation $(h)^{*}$ & $11(8-14)$ & $14(7-18)$ & .53 \\
\hline Duration of ICU therapy (d)* & $2(2-3)$ & $6(4-8)$ & $<.01$ \\
\hline Length of hospital stay $(d)^{*}$ & $15(14-22)$ & $18(15-27)$ & .17 \\
\hline Maximum epinephrine dosage $(\mathrm{mg} / \mathrm{h})^{*}$ & $0.0(0.0-0.2)$ & $0.0(0.0-0.2)$ & .60 \\
\hline Duration of epinephrine therapy $(d)^{*}$ & $0.0(0.0-0.5)$ & $0.0(0.0-1.0)$ & .20 \\
\hline Maximum norepinephrine dosage $(\mathrm{mg} / \mathrm{h})^{*}$ & $0.5(0.3-1.0)$ & $0.8(0.6-1.7)$ & .02 \\
\hline Duration of norepinephrine therapy (d)* & $1.0(1.0-2.0)$ & $4.0(2.0-4.5)$ & $<.01$ \\
\hline Lactate $(\mathrm{mmol} / \mathrm{L})^{*} \ddagger$ & $2.3(2-3.4)$ & $2.6(2-4.5)$ & .47 \\
\hline Interleukin $6(\mathrm{pg} / \mathrm{mL})^{*} \S$ & $82(56-208)$ & 309 (181-439) & .05 \\
\hline
\end{tabular}

SAPS II, Simplified Acute Physiology Score; TISS, Therapeutic Intervention Scoring System; CPB, cardiopulmonary bypass; ICU, intensive care unit. *Data are presented as medians (25th-75th percentiles). †Indicators of disease severity (for definition, see Table E1). $\ddagger$ Peak concentration measured during intensive care unit treatment. §Measured at 48 hours after admittance to the intensive care unit.

matic memories, and chronic stress symptoms. The completed questionnaires were mailed back by the patients.

\section{Instruments and Key Measures of the Study End Points}

HRQL. HRQL at 6 months after discharge from the ICU was measured by using the self-administered Medical Outcomes Study Short Form Survey that consists of 36 questions (SF-36). The SF-36 covers 8 basic health concepts and has been widely used for HRQL outcome evaluation of CS. ${ }^{15}$

Chronic stress symptoms and PTSD. The severity of stress symptoms as they existed at 6 months after CS was measured with a modified version of the Posttraumatic Stress Symptom 10 Question Inventory (PTSS-10) questionnaire, which has been validated in patients after ICU treatment. ${ }^{16}$ This instrument records the presence and intensity of 10 stress symptoms: (1) sleep disturbance, (2) nightmares, (3) depression, (4) hyperalertness, (5) withdrawal (emotional numbing and inability to care for others), (6) generalized irritability, (7) frequent changes in mood, (8) guilt, (9) fear and avoidance reactions with regard to the ICU, and (10) increased muscle tension. Patients rated their symptoms on a scale from 1 (never) to 7 (always). A summary score of more than 35 is associated with a high probability of patients fulfilling the diagnostic criteria for PTSD. ${ }^{16}$

Evaluation of traumatic memories. All patients were asked to complete a structured and validated questionnaire, evaluating different categories of traumatic memory from ICU therapy ${ }^{16}{ }^{\text {For the }}$ purpose of this study, a category of traumatic memory as measured by the questionnaire was defined as the patients' subjective recollection of (1) respiratory distress-dyspnea, (2) feelings of anxietypanic, (3) pain, or (4) nightmares from the ICU. ${ }^{16}$ The patients were asked to answer each of these 4 items "yes" or "no," independent of the number of occasions the adverse experience oc- curred. The number of items answered "yes" by a subject was termed the number of categories of traumatic memory present.

In addition, when completing the questionnaire, patients were asked to describe earlier and preexisting traumatic experiences (eg, from the war) unrelated to CS and ICU therapy.

\section{Statistics}

Because in our previous studies neither stress symptoms nor SF-36 HRQL outcome scores were normally distributed, ${ }^{5,11}$ we intended to use nonparametric tests. Continuous variables between the hydrocortisone and placebo groups were compared with the MannWhitney $U$ test. Discrete variables were compared with the $\chi^{2}$ or Fisher exact tests, where appropriate. Spearman rho was calculated as a nonparametric measure of linear association between variables (eg, the duration of ICU therapy and scores on the stress symptom inventory). All statistical tests were of an exploratory nature. Statistical calculations were performed with the SPSS 12.01 statistical package (SPSS Inc, Chicago, Ill). Results are expressed as medians and 25 th to 75 th percentiles.

\section{Results}

Of the 36 patients enrolled in the study, 20 had undergone coronary artery bypass grafting, 7 had undergone valve operations, and 9 had undergone both procedures. From these 36 patients, 30 returned questionnaires at 6 months after CS; 2 of these patients had to be excluded from the final analyses because of incomplete questionnaires (missing data on traumatic memories and stress symptom scores). This resulted in a final participation rate of $78 \%$ ( 28 patients). Five of the excluded patients were from the hydrocortisone group, and 3 were from the placebo group $(P=.70$, Fisher exact 
TABLE 2. Comparison of SF-36 HROL scores at 6 months after CS between patients from the hydrocortisone and placebo groups

\begin{tabular}{lccc}
\hline SF-36 items* & Hydrocortisone group $(\mathbf{n}=\mathbf{1 4})$ & Placebo group $(\mathbf{n}=\mathbf{1 4})$ & \multicolumn{1}{c}{$\boldsymbol{P}$ value } \\
\hline Physical function & $85(49-90)$ & $38(35-60)$ & .01 \\
Physical role function & $25(0-75)$ & $0(0-50)$ & .19 \\
Paint & $100(72-100)$ & $62(36-88)$ & .01 \\
General health perception & $72(65-75)$ & $60(49-63)$ & 4.01 \\
Vitality & $58(44-76)$ & $40(29-46)$ & $<.01$ \\
Social function & $88(75-100)$ & $69(50-81)$ & .06 \\
Emotional role function & $67(17-100)$ & $0(0-67)$ & .10 \\
Mental health & $80(66-84)$ & $64(51-69)$ & .01
\end{tabular}

SF-36, Short Form-36; HROL, health-related quality of life; CS, cardiac surgery. *Data are presented as medians (25th-75th percentiles). $†$ Note: SF-36 pain scores show a reverse coding (eg, higher scores indicate a lower pain incidence and intensity).

test). There were no significant differences with regard to patient or treatment characteristics between included or excluded patients (Table E1).

\section{Demographic and Treatment Characteristics of the Study Population}

Patients from the hydrocortisone and placebo groups did not differ with regard to the type of surgical procedures (data not shown), age and sex distribution, or the duration of $\mathrm{CPB}$ or aortic crossclamping (Table 1). Compared with patients from the placebo group, however, patients who received hydrocortisone had a significantly shorter postoperative stay in the ICU, had significantly lower TISS scores, showed a strong trend toward lower values of the proinflammatory cytokine IL-6 $(P=.05)$, and required significantly less norepinephrine (both with regard to maximal dosage and duration of administration, Table 1). When all 8 patients with missing long-term outcome data were included in the short-term outcome analyses, this had no effect on overall short-term results; patients from the hydrocortisone group still had significantly lower norepinephrine requirements (regarding dosage and duration of exposure), a significantly shorter duration of ICU stay ( 2 vs 6 days, median values; $P<.01$ ), and significantly lower TISS scores (Table E2).

\section{HRQL Outcomes}

At 6 months after CS, patients from the hydrocortisone group had higher scores on 7 of 8 scales of the SF-36 that comprised both physical and mental HRQL aspects, with significant differences regarding physical function, pain, general health perception, vitality, and emotional well-being (Table 2) and no improvement regarding physical role function. Likewise, patients from the hydrocortisone group had significantly higher SF-36 physical and mental summary scores compared with those of patients from the placebo group (Figure 1). When the 2 patients excluded from the stress symptom analyses because of missing data were included in the HRQL comparison, this did not significantly change the results (data not shown).

\section{Chronic Stress Symptoms and PTSD}

Patients from the hydrocortisone group had significantly lower chronic stress symptom scores when compared with those of patients from the placebo group (median of 15.5 [interquartile range, 14.8-21.8] in the hydrocortisone group vs median of 25.5 [interquartile range, 16.8-33.0] in the control subjects, $P=.03$ ). Stress symptom scores correlated positively with the duration of ICU therapy (Spearman rho $=0.38, P=.05$ ). There were significant but not very high correlations between stress symptom scores and SF-36 physical pain (rho $=-0.45, P=.02$ ), vitality (rho $=$ $-0.48, P=.01$ ), social function (rho $=-0.50, P<.01$ ),

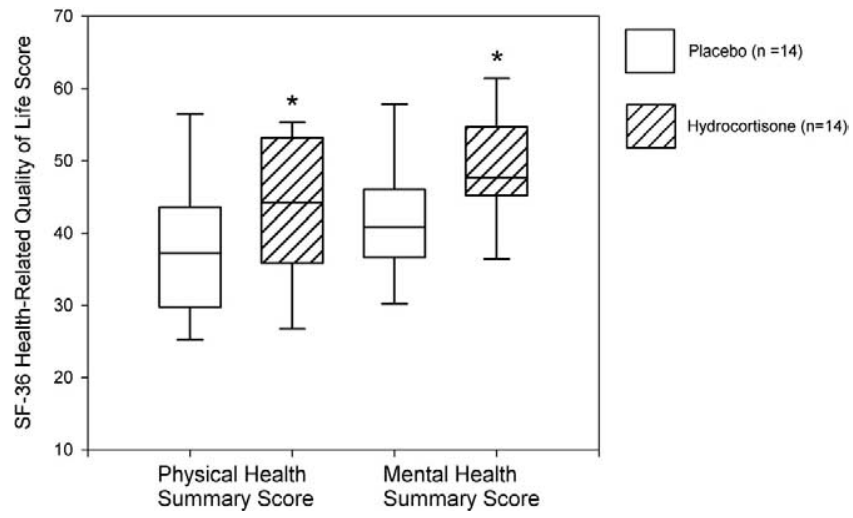

Figure 1. Comparison of Short Form-36 health-related quality-oflife summary scores between patients from the hydrocortisone group ( $n=14$, shaded box plots) and patients from the placebo group ( $n=14$, open box plots) measured at 6 months after cardiac surgery. The whiskers at the top and bottom of each box indicate the 5th and 95th percentiles of the distribution, respectively; the top and bottom of each box indicate the 75th and 25th percentiles, respectively; and the line through the box indicates the median (the 50th percentile). ${ }^{*} P \leq .03$. 
mental health (rho $=-0.60, P<.01$ ), and mental health summary scores (rho $=-0.60, P<.01$ ).

Three of 14 patients from the placebo group and 1 of 14 patients from the hydrocortisone group showed evidence of PTSD (as defined as a stress symptom score $>35$ points $^{16}$ ).

\section{Categories of Traumatic Memory}

With regard to the total study population, $15(53.6 \%)$ patients had potentially traumatic memories from the cardiovascular ICU, whereas 13 (46.4\%) did not recall aversive experiences. The most common category of traumatic memory from ICU treatment in the total study population was the recall of respiratory distress in $6(21.4 \%)$ patients, followed by memories of pain in 5 (17.9\%) patients, nightmareshallucinations in another 5 patients, and anxiety-panic in 3 $(10.7 \%)$ individuals. Patients from the hydrocortisone and placebo groups did not differ significantly with regard to the number and type of traumatic memories $(P \leq .33)$.

Twelve $(42.9 \%)$ patients reported preexisting traumatic memories unrelated to cardiovascular disease (mostly war related). The number of patients with and without preexisting traumatic memories did not differ between groups $(P=$ .70). Patients with preexisting traumatic memories demonstrated an increased risk for traumatic memories from the cardiovascular ICU (9/12 patients with preexisting traumatic memories also had at least one traumatic memory from the ICU compared with $6 / 16$ patients without prior trauma, $P=.07$ ).

\section{Discussion}

This pilot study shows that stress doses of hydrocortisone given to a predefined group of high-risk cardiac surgical patients at induction of anesthesia and continued during ICU treatment resulted in considerable short- and long-term effects. Short-term advantages of stress doses of hydrocortisone found in this study were a significant shortening of the duration of ICU treatment, lower TISS scores, reduced norepinephrine requirements, and a strong trend toward lower concentrations of the proinflammatory cytokine IL-6. These findings are in agreement with those of a previously performed study in a comparable high-risk group of patients, which evaluated only the short-term benefits of stress doses of hydrocortisone during CS. ${ }^{13}$

The long-term benefits of hydrocortisone treatment found in this trial were a reduced incidence and intensity of chronic stress symptoms and improvements in overall HRQL outcomes. Importantly, these health benefits included improvements in both mental and physical aspects of HRQL. In this regard it is of interest to note that the occurrence of PTSD symptoms in survivors of myocardial infarction significantly increases the likelihood of cardiovascular readmission and a negative outcome. ${ }^{17}$ The inverse relationship between the presence of chronic stress symptoms and
HRQL has been demonstrated in this study, as well as in other patients after $\mathrm{CS},{ }^{3,5}$ critical illness requiring prolonged ICU therapy, ${ }^{18,19}$ or other types of massive stressors. ${ }^{20}$ Whereas the negative relationship between the occurrence of PTSD symptoms and mental health aspects of HRQL appears to be conceivable, negative effects of chronic stress symptoms on physical function in this and another study ${ }^{3}$ are more difficult to explain. One could speculate, however, that patients with a high incidence and intensity of stress symptoms are unable to appreciate possible physical benefits of CS because stress symptoms might be perceived as the constant presence of physical disease related to the underlying coronary heart disease. In addition, the continued presence of stress symptoms could result in an increase in allostatic load, ${ }^{21}$ changes in immune function, and noncompliance with medication, ${ }^{17}$ which could all contribute to adverse physical outcomes.

It is therefore likely that at least some of the long-term effects of hydrocortisone on HRQL outcomes found in this study are caused by a limitation and downregulation of the original stress response triggered during the perioperative phase of CS. ${ }^{3}$ Increased stress exposure in patients from the placebo group might be reflected by higher norepinephrine requirements found in this and another study, ${ }^{13}$ although the number and type of traumatic memories (another important indicator of perioperative $\operatorname{stress}^{22}$ ) did not differ between the groups.

Glucocorticoids have also been reported to directly affect the recall of stored information. Some of the long-term effects of hydrocortisone treatment on chronic stress symptoms (and likely HRQL) might have been mediated by these well-established effects of glucocorticoids on mnemonic processes. ${ }^{23}$ In this regard it is of interest to note that patients found to have a low urinary cortisol excretion immediately after a car accident had a higher incidence of intrusive memories from the accident. ${ }^{24}$ Furthermore, longterm survivors of the acute respiratory distress syndrome with a high number of traumatic memories from ICU therapy showed significantly lower serum cortisol levels, together with an increased incidence of chronic stress symptoms and risk for PTSD (unpublished data). Because it has been proposed that PTSD symptoms develop over time after trauma because of positive feedback mechanisms in which the traumatic memories are constantly retrieved and restored, ${ }^{25}$ low serum cortisol levels could facilitate repeated recollections of the traumatic experiences, resulting in strong intrusive memories and PTSD. A disruption of retrieval mechanisms in a close temporal relationship with a traumatic experience caused by exogenously administered hydrocortisone could theoretically result in a long-term reduced incidence or intensity of PTSD symptoms. ${ }^{26}$ These speculations are corroborated by findings from a pilot study that prolonged administration of a very low dose of hydro- 
cortisone administered to patients with preexisting PTSD of nonmedical origin resulted in a significant reduction of traumatic memory retrieval. ${ }^{27}$

The issue of side effects from the treatment with hydrocortisone has not been addressed by this study because the number of patients was too small to detect these effects. However, Kilger and colleagues ${ }^{13}$ could demonstrate that hydrocortisone, administered in the described way, does not go along with relevant side effects, including acid-base disorders and wound infections, in a larger cohort of patients.

In summary, our study indicates that the administration of hydrocortisone in a dosage comparable with the endogenous maximal production rate during the perioperative phase of CS is associated with a lower incidence of chronic stress symptoms and an improvement in HRQL in selected patients at high risk for perioperative complications. These findings might have important implications regarding both economic and pathophysiologic aspects of outcome in CS but need to be confirmed in larger randomized trials.

We thank Dr James L. McGaugh for valuable comments on an earlier version of the manuscript.

\section{References}

1. Hunt JO, Hendrata M, Myles PS. Quality of life after cardiac surgery. Heart Lung. 2000;29:401-11.

2. Newman MF, Kirchner JL, Phillips-Bute B, Gaver V, Grocott H, Jones $\mathrm{RH}$, et al. Longitudinal assessment of neurocognitive function after coronary-artery bypass surgery. $N$ Engl J Med. 2001;344:395-402.

3. Schelling G, Richter M, Roozendaal B, Rothenhausler H, Stoll C, Nollert G, et al. Exposure to high stress in the ICU may have negative effects on health-related quality of life outcomes after cardiac surgery. Crit Care Med. 2003;31:1971-80.

4. Rothenhausler HB, Grieser B, Nollert G, Reichart B, Schelling G, Kapfhammer HP. Psychiatric and psychosocial outcome of cardiac surgery with cardiopulmonary bypass: a prospective 12-month follow-up study. Gen Hosp Psychiatry. 2005;27:18-28.

5. Stoll C, Schelling G, Goetz AE, Kilger E, Bayer A, Kapfhammer HP, et al. Health-related quality of life and post-traumatic stress disorder in patients after cardiac surgery and intensive care treatment. $J$ Thorac Cardiovasc Surg. 2000;120:505-12.

6. Yehuda R. Post-traumatic stress disorder. N Engl J Med. 2002;346: 108-14.

7. Doerfler LA, Pbert L, DeCosimo D. Symptoms of posttraumatic stress disorder following myocardial infarction and coronary artery bypass surgery. Gen Hosp Psychiatry. 1994;16:193-9.

8. Yehuda R. Current status of cortisol findings in post-traumatic stress disorder. Psychiatr Clin North Am. 2002;25:341-68, vii.

9. Neylan TC, Brunet A, Pole N, Best SR, Metzler TJ, Yehuda R, et al. PTSD symptoms predict waking salivary cortisol levels in police officers. Psychoneuroendocrinology. 2005;30:373-81.
10. Schelling G, Briegel J, Roozendaal B, Stoll C, Rothenhausler HB, Kapfhammer HP. The effect of stress doses of hydrocortisone during septic shock on posttraumatic stress disorder in survivors. Biol Psychiatry. 2001;50:978-85.

11. Schelling G, Kilger E, Roozendaal B, Briegel J, Dagge A, Rothenhausler $\mathrm{HB}$, et al. Stress doses of hydrocortisone, traumatic memories and post-traumatic stress disorder in patients after cardiac surgery: a randomized study. Biol Psychiatry. 2004;55:627-33.

12. Briegel J, Forst F, Haller M, Schelling G, Kilger E, Kuprat G, et al. Stress doses of hydrocortisone reverse hyperdynamic septic shock: a prospective, randomized, double-blind, single center study. Crit Care Med. 1999;27:723-32.

13. Kilger E, Weis F, Briegel J, Frey L, Goetz A, Reuter D, et al. Stress doses of hydrocortisone reduce severe systemic inflammatory response syndrome and improve early outcome in a risk group of patients after cardiac surgery. Crit Care Med. 2003;31:1068-74.

14. McGaugh JL, Roozendaal B. Role of adrenal stress hormones in forming lasting memories in the brain. Curr Opin Neurobiol. 2002; $12: 205-10$

15. Ware JE, Sherbourne CD. The MOS 36-Item Short-Form Health Survey (SF-36): I. Conceptual framework and item selection. Med Care. 1992;30:473-81.

16. Stoll C, Kapfhammer HP, Haller H, Briegel J, Krauseneck T, Durst K, et al. Sensitivity and specificity of a screening test to document traumatic experiences and to diagnose post-traumatic stress disorder in patients after intensive care treatment. Intensive Care Med. 1999;25: 697-704.

17. Shemesh E, Yehuda R, Milo O, Dinur I, Rudnick A, Vered Z, et al. Posttraumatic stress, nonadherence, and adverse outcome in survivors of a myocardial infarction. Psychosom Med. 2004;66:521-6.

18. Kapfhammer HP, Rothenhausler HB, Krauseneck T, Stoll C, Schelling G. Posttraumatic stress disorder and health-related quality of life in long-term survivors of acute respiratory distress syndrome. Am J Psychiatry. 2004;161:45-52.

19. Schelling G, Stoll C, Kapfhammer HP, Rothenhausler HB, Krauseneck $\mathrm{T}$, Durst K, et al. The effect of stress doses of hydrocortisone during septic shock on posttraumatic stress disorder and health-related quality of life in survivors. Crit Care Med. 1999;27:2678-83.

20. d'Ardenne P, Capuzzo N, Fakhoury WK, Jankovic-Gavrilovic J, Priebe S. Subjective quality of life and posttraumatic stress disorder. J Nerv Ment Dis. 2005;193:62-5.

21. McEwen BS. Mood disorders and allostatic load. Biol Psychiatry. 2003;54:200-7.

22. Schelling G. Effects of stress hormones on traumatic memory formation and the development of posttraumatic stress disorder in critically ill patients. Neurobiol Learn Mem. 2002;78:596-609.

23. de Quervain DJ, Roozendaal B, Nitsch RM, McGaugh JL, Hock C. Acute cortisone administration impairs retrieval of long-term declarative memory in humans. Nat Neurosci. 2000;3:313-4.

24. Delahanty DL, Raimonde AJ, Spoonster E. Initial posttraumatic urinary cortisol levels predict subsequent PTSD symptoms in motor vehicle accident victims. Biol Psychiatry. 2000;48:940-7.

25. Pitman RK, Orr SP, Shalev AY. Once bitten, twice shy: beyond the conditioning model of PTSD. Biol Psychiatry. 1993;33:145-6.

26. Schelling G, Roozendaal B, de Quervain DJ-F. Can post-traumatic stress disorder (PTSD) be prevented with glucocorticoids? Ann N Y Acad Sci. 2004;1032:158-66.

27. Aerni A, Traber A, Hock C, Roozendaal B, Schelling G, Papassotiropoulos A, et al. Low-dose cortisol treatment reduces symptoms of post-traumatic stress disorder. Am J Psychiatry. 2004;161:1488-90. 
TABLE E1. Comparison of patient characteristics and treatment data between the final study population (included patients) and patients who were originally randomized into the study $(n=36)$ but did not return questionnaires $(n=6)$ or had to be excluded because of incomplete questionnaires $(n=2)$

\begin{tabular}{|c|c|c|c|}
\hline Patient and treatment data & Included patients $(\mathbf{n}=\mathbf{2 8})$ & Excluded patients $(n=8)$ & $P$ value \\
\hline $\operatorname{Sex}(F / M)$ & $6 / 22$ & $2 / 6$ & 1.00 \\
\hline Age $(y)^{*}$ & $69(61-71)$ & $68(67-79)$ & .38 \\
\hline SAPS II*† & $36(30-38)$ & $38(31-57)$ & .40 \\
\hline TISS* $\ddagger$ & $116(84-246)$ & $145(112-255)$ & .64 \\
\hline Preoperative left ventricular ejection fraction (\%) & $30(26-43)$ & $32(22-38)$ & .82 \\
\hline Duration of CPB $(\mathrm{min})^{*}$ & $117(99-147)$ & $105(100-166)$ & .17 \\
\hline Duration of aortic crossclamp $(\mathrm{min})^{*}$ & $72(60-105)$ & $81(77-112)$ & .21 \\
\hline Duration of mechanical ventilation $(\mathrm{h})^{*}$ & $11(7-16)$ & $12(9-16)$ & .67 \\
\hline Duration of ICU therapy $(\mathrm{d})^{*}$ & $3(2-4)$ & $4(2-6)$ & .87 \\
\hline Length of hospital stay $(d)^{*}$ & $17(14-22)$ & $16(14-28)$ & .84 \\
\hline Maximum epinephrine dosage $(\mathrm{mg} / \mathrm{h})^{*}$ & $0.0(0.0-0.2)$ & $0.1(0.0-0.3)$ & .38 \\
\hline Duration of epinephrine therapy $(d)^{*}$ & $0.0(0.0-0.5)$ & $0.3(0.0-1.0)$ & .34 \\
\hline Maximum norepinephrine dosage $(\mathrm{mg} / \mathrm{h})^{*}$ & $0.6(0.5-1.3)$ & $0.9(0.4-1.0)$ & .75 \\
\hline Duration of norepinephrine therapy $(d)^{*}$ & $1.8(1.0-3.8)$ & $2.5(1.0-4.0)$ & .67 \\
\hline Lactate $(\mathrm{mmol} / \mathrm{L})^{*} \S$ & $2.6(1.8-3.9)$ & $2.7(2.2-6.6)$ & .47 \\
\hline Interleukin $6(\mathrm{pg} / \mathrm{mL})^{*} \|$ & $169(73-254)$ & $309(71-230)$ & .57 \\
\hline
\end{tabular}

$C P B$, Cardiopulmonary bypass; ICU, intensive care unit. *Data are presented as medians (25th-75th percentiles). $†$ SAPS II (Simplified Acute Physiology Score II) provides an estimate of the risk of death in the intensive care unit without having to specify a primary diagnosis and includes 17 variables: 12 physiology variables, age, type of admission (scheduled surgical, unscheduled surgical, or medical), and 3 underlying disease variables (AIDS, metastatic cancer, and hematologic malignancy). The SAPS II has also been validated in patients from coronary care units. SAPS II was measured in the morning of postoperative day 1. $\$$ TISS (Simplified Therapeutic Intervention Scoring System) provides an estimate of the time spent on nursing activities in a given patient (1 TISS point is approximately equal to 11 minutes of nursing activities). §Peak concentration measured during intensive care unit treatment. ||Measured at 48 hours after admittance to the intensive care unit.

TABLE E2. A comparison of patient characteristics and short-term outcomes between patients from the hydrocortisone and placebo groups

\begin{tabular}{|c|c|c|c|}
\hline Patient and treatment data & Hydrocortisone group ( $n=19$ ) & Placebo group $(n=17)$ & $P$ value \\
\hline $\operatorname{Sex}(F / M)$ & $4 / 15$ & $4 / 13$ & .86 \\
\hline Age $(y)^{*}$ & $68(63-72)$ & $69(63-73)$ & .69 \\
\hline SAPS $1 I^{*} \dagger$ & $34(30-38)$ & $36(31-46)$ & .51 \\
\hline TISS* $\ddagger$ & $99(78-129)$ & $234(149-356)$ & $<.01$ \\
\hline Preoperative left ventricular ejection fraction (\%) & $30(26-39)$ & $31(21-49)$ & .84 \\
\hline Duration of CPB $(\mathrm{min})^{*}$ & $98(91-154)$ & $112(65-139)$ & .64 \\
\hline Duration of aortic crossclamp $(\mathrm{min})^{*}$ & $85(43-113)$ & $75(62-86)$ & .85 \\
\hline Duration of mechanical ventilation $(\mathrm{h})^{*}$ & $11(8-14)$ & $14(7-18)$ & .53 \\
\hline Duration of ICU therapy $(d)^{*}$ & $2(2-3)$ & $6(4-8)$ & $<.01$ \\
\hline Length of hospital stay $(d)^{*}$ & $15(14-22)$ & $18(15-27)$ & .19 \\
\hline Maximum norepinephrine dosage $(\mathrm{mg} / \mathrm{h})^{*}$ & $0.5(0.3-1.0)$ & $0.8(0.6-1.7)$ & .02 \\
\hline Duration of norepinephrine therapy $(d)^{*}$ & $1.0(1.0-2.0)$ & $4.0(2.0-4.5)$ & $<.01$ \\
\hline Lactate $(\mathrm{mmol} / \mathrm{L})^{*} \S$ & $2.3(2.0-3.4)$ & $2.6(2.0-4.5)$ & .47 \\
\hline Interleukin $6(\mathrm{pg} / \mathrm{mL})^{*} \|$ & $82(56-207)$ & $309(181-440)$ & .05 \\
\hline
\end{tabular}

This comparison includes all originally randomized patients $(n=36)$. CPB, Cardiopulmonary bypass; ICU, intensive care unit. *Data are presented as medians (25th-75th percentiles). $†$ The SAPS II (Simplified Acute Physiology Score II) provides an estimate of the risk of death in the intensive care unit without having to specify a primary diagnosis and includes 17 variables: 12 physiology variables, age, type of admission (scheduled surgical, unscheduled surgical, or medical), and 3 underlying disease variables (AIDS, metastatic cancer, and hematologic malignancy). The SAPS II has also been validated in patients from coronary care units. SAPS II was measured in the morning of postoperative day 1. $\ddagger$ TISS (Simplified Therapeutic Intervention Scoring System) gives an estimate of the time spent on nursing activities in a given patient (1 TISS point is approximately equal to 11 minutes of nursing activities). §Peak concentration measured during intensive care unit treatment. |Measured at 48 hours after admittance to the intensive care unit. 